\title{
Local Quantum Physics and Models
}

\author{
Rudolf Haag ${ }^{\star}$ \\ Research Institute for Mathematical Sciences, Kyoto University, Kyoto 606, Japan
}

Received September 17, 1992

Dedicated to Huzihiro Araki

Abstract. The problem of characterizing a specific model within the frame of local quantum physics is addressed.

\section{Introduction}

A round birthday provides an occasion to take stock, to look back at questions which puzzled us years ago and to the answers arrived at, but more importantly, to recognize unsatisfactory features and problems ahead. We are concerned here with the synthesis of quantum theory with the principle of locality on the level of special relativity, in short with Local Quantum Physics, an area to which Huzihiro Araki contributed so much. Let us briefly sketch the ingredients.

1) We accept space-time as described in special relativity. It is the 4-dimensional Minkowski space $\mathscr{C} b$ equipped with its a priori given causal and metric structure. Its symmetry group is the Poincare group $\mathscr{P}$ generated by translations and Lorentz transformations.

2) From quantum theory we take the notions of "observables" and "states." The former may be materialized by measuring equipment, the latter define probabilities for the outcome of measurements. Observables are mathematically represented by selfadjoint elements of a non-commutative algebra, states by normalized positive linear forms on this algebra.

3) The principle of locality demands that we order the observables according to the regions in space-time to which they refer. We use the symbol (9) to denote a contractible open subset of $\mathscr{C l}$, with compact closure (a finitely extended region). The subalgebra generated by all observables in $\odot$ is denoted by $\mathfrak{A}(\odot)$. Einstein causality demands that the algebras $\mathfrak{A}\left(Q_{1}\right)$ and $\mathfrak{A}\left(\mathscr{Q}_{2}\right)$ commute whenever $\mathscr{O}_{1}$ is space-like to $\mathscr{O}_{2}$. Poincare symmetry demands that there is a realization of $\mathscr{P}$ by automorphisms of the

* Permanent address: Inst. f. Theoret. Phys., Univ. Hamburg, Luruper Chaussee 149, D-22761 Hamburg, Germany 
"net of algebras" $\{\mathfrak{A}(\mathscr{O})\}$. Thus to every element $g \in \mathscr{P}$ we have an automorphism $\alpha_{g}$ with

$$
\alpha_{g} \mathfrak{A}(\odot)=\mathfrak{A}(g \odot) .
$$

It suffices to consider bounded observables and correspondingly the algebras are assumed to be $C^{*}$-algebras. One has the obvious inclusion relations of the algebras corresponding to the inclusion of regions. This allows the definitions of the "algebra of all local observables" as the union of all $\mathfrak{A}(\mathcal{O})$ and the "algebra of all quasilocal observables," denoted by $\mathfrak{A}$ as the completion in the norm topology of the former. We should keep in mind, however, that the physical information rests in the subdivision of $\mathfrak{A}$ into the distinguished family of subalgebras $\mathfrak{A}(\mathscr{\odot})$, i.e. in the "net," not in the total algebra $\mathfrak{A}$ which is only a construct for convenience of language.

The next step is to realize that the algebras $\mathfrak{A}(\mathcal{G})$ should be considered as abstract algebras, defined by their internal algebraic relations, and not as operator algebras acting on a Hilbert space. This implies that we may expect the existence of inequivalent representations of the net by a net of operator algebras and this feature is used to explain superselection rules. Each equivalence class of representations has its own family of states, its "folium of states." These are the states which can be described by state vectors or density matrices in the Hilbert space on which the representation acts. For inequivalent primary representations these folia are disjoint. Each folium corresponds to one superselection sector.

4) Stability (Principle of positive energy). Poincaré symmetry allows us to control the transfer of energy, momentum, angular momentum etc. induced by the action of algebraic elements on states. Energy itself, as a global quantity, is not associated to the local algebras. We have to think of energy density. Stability means, roughly speaking, that the energy content of a finite region has a lower bound. There are two types of representation classes with which we are dealing. In the first type, the total energy and momentum can be defined. For this type there is a canonical determination of the zero-point of energy momentum so that energies can be compared also for states in different superselection sectors of this type. The set of representation classes of this

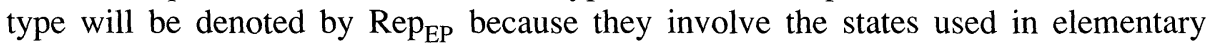
particle physics. For these representations the stability requirement is sharpened by demanding that the joint spectrum of energy and momentum is confined to the forward cone (positive energy, real mass). Usually one assumes also that there is a unique (normalized) state of zero energy, the vacuum. The other type, denoted by $\operatorname{Rep}_{\mathrm{H}}$, describes states with nonvanishing energy density extending up to infinity. These may be called hydrodynamic states and among these the thermodynamic equilibrium states are of special interest. The total energy and energy fluctuation in these states is infinite.

Of course, Local Quantum Physics has grown out from Quantum Field Theory. So we should compare the parallels and differences of input and philosophy. In Quantum Field Theory we start with field quantities labelled by points in space-time. Among them are observable fields and others which are in principle unobservable. This arises because in its origin Quantum Field Theory relied strongly on a fieldparticle duality: to every species of elementary particle one associates a field and for every basic field one assumes a corresponding particle type. There are particles obeying Fermi statistics with charge quantum numbers of different kind. Observables do not connect states with different charge and they commute at space-line distances due to Einstein causality. Therefore in the picture of field-particle duality unobservable fields serve to describe the charge structure. The commutation relations of the fields mirror the statistics of the associated particles. The physical interpretation hinges then 
on an "asymptotic condition" relating the creation operators of particles in incoming or outgoing configurations to limits of field operators at large negative or positive times. In Local Quantum Physics the physical interpretation is entirely delegated to the space-time geometric placement of observables and it is claimed that once the correspondence

$$
\text { ๑ } \rightarrow \mathfrak{A}(\odot)
$$

is given (satisfying the above mentioned conditions) the theory is completely defined and all information about particles etc. can be extracted. To verify this claim one has the task of describing how the information about particle types, collision cross sections, charges, exchange symmetry (statistics) is encoded in the net of observable algebras. This has been achieved in a lengthy endeavour by a relatively small group of authors and resulted in a deeper understanding of the structure of the theory. All this concerns the states in $\operatorname{Rep}_{\mathrm{EP}}$. The discussion of the other states in $\operatorname{Rep}_{\mathrm{H}}$ leads to a natural description of equilibrium states in the thermodynamic limit, simpler than that for a system enclosed in a box, invoking the notions of dynamical stability and passivity and exhibiting a remarkable connection to the mathematical theory of modular automorphisms of algebras.

Here is not the place to describe this work. I have done this in [1].

\section{Shortcomings and their Possible Mending}

Looking at the frame described thus far and at its elaborations we can satisfied to have a consistent scheme based on a few clear principles and leading to a remarkably rich structure of consequences in agreement with experience. There are, however, several unsatisfactory points.

1) While we can claim that a specific theory including its physical interpretation is characterized as soon as we have given the correspondence (2) such that it meets the listed requirements, we have no method of constructing this correspondence without starting from a specific field theoretical model and there has been no attempt to classify the theories within the general frame.

2) The notion of observables which refer to specific regions of space-time is unproblematic if we think of regions with a radius large than $10^{-8} \mathrm{~cm}$. It becomes a highly questionable idealization for regions of radius below $10^{-13} \mathrm{~cm}$. Yet the qualitative agreement of high energy experiments with models in Quantum Field Theory indicates that the principle of relativistic locality remains valid down to distances of order $10^{-16} \mathrm{~cm}$ and presumably even below that. This suggests that the notion of "local observable" should better be replaced by that of "localized event."

3) All superselection rules which appear to play a rôle in physics refer to the whole net while for an individual local algebra $\mathfrak{A}(\odot)$ there seems to be only one physically allowed representation class, irrespective of whether we consider states in $\operatorname{Rep}_{\mathrm{EP}}$ or in $\operatorname{Rep}_{\mathrm{H}}$. If we regard $\mathfrak{A}(\odot)$ as an abstract algebra this feature needs an explanation. This is related to the somewhat unsatisfactory formulation of the stability principle, most clearly seen if we want to go over to quantum field theory in curved space-time when we do not have the Poincaré automorphisms. What is needed is a restriction on the class of physically allowed states. Not all positive linear forms of the algebra are physically realizable as states.

4) The reason why observables should be elements of a non-commutative algebra remains (almost) as dark as in the early days of quantum mechanics. 
All these critical remarks point in one direction: We must focus more attention on the state space. Its structure is presumably more basic than the algebras considered above.

State space is naturally embedded in a so-called "base norm space." This is a real linear space $V$ with a distinguished convex cone $V^{+}$and a norm which coincides on $V^{+}$with the value taken by a distinguished positive linear form $e$ on $V$. So much follows directly from the aspect of states as summarizing probability predictions for future events. In particular the real linearity in $V$ comes from the possibility of forming convex combination of states (the mixing process). The specific quantum theoretical restrictions concern the structure of "faces" of the cone $V^{+}$. A face $F$ is a convex subcone of $V^{+}$with the property that if $\varphi \in F$ and $\varphi=\lambda_{1} \varphi_{1}+\lambda_{2} \varphi_{2}$ with $\lambda_{1}, \lambda_{2}>0$ then also $\varphi_{1}$ and $\varphi_{2} \in F$. A face is part of the boundary of $V^{+}$; a 1 dimensional face is a pure state. In Quantum Mechanics $V$ consists of the self adjoint trace-class operators on Hilbert space; a face corresponds to the set of self-adjoint trace class operators on a subspace. The dual space $V^{*}$ of $V$ (the set of real linear forms on $V$ ) is the set of observables. If we start from the states rather than from the observables we should consider an observable $A$ as a linear form on state space (on $V$ ) rather than vice versa and write the expectation value of $A$ is a state $\omega$ as $A(\omega)$ instead of $\omega(A)$. The linear structure of the set of observables and the distinction of a convex cone $V^{*(+)}$ (positive observables) is then naturally inherited from $V$. The product (or rather Jordan product) between observables has no such evident origin. A product appears naturally if we consider operations on states, i.e. mappings of $V$ onto (or into) $V$ conserving the structure but we shall not pursue the relation of this to the algebraic structure of the set of observables here. There is only one feature of the quantum theoretic formalism used in the subsequent discussion: to a face $F$ in $V^{+}$there corresponds a unique element in $V^{*(+)}$, its "subunit" $E_{F}$. It is a positive observable with unit norm having its maximal value on the face $F$ and assuming its minimal value 0 on a complementary face $F^{\prime}$. In Quantum Mechanics it corresponds to the projection operator on the subspace characterizing the face. We shall call $F$ or $e_{F}$ a "potential event."

What about locality? Obviously any state may be restricted to the observables or events within a region 9 . Thus, for every $C$, we have a set of "partial states" $\mathscr{S}(\bigcirc)$ which generate again a base norm space $V(\odot)$. An element of $\mathscr{P}(\circlearrowleft)$ is an equivalence class of states on larger regions, the equivalence relation being

$$
\omega_{1} \sim \omega_{2} \quad \text { if }\left.\quad \omega_{1}\right|_{\mathfrak{A}_{(\varsigma)}}=\left.\omega_{2}\right|_{\mathfrak{A}_{(\varsigma)}} .
$$

This may be continued to define equivalence classes with respect to a single point in Minkowski space: $\omega_{1} \underset{x}{\sim} \omega_{2}$ if there exists some open neighbourhood of $x$ on which $\omega_{1}$ and $\omega_{2}$ coincide.

The collection of sets $\mathscr{S}(\mathscr{O})$ is a presheaf with the canonical restriction map leading from $\mathscr{S}\left(\mathscr{O}_{1}\right)$ to $\mathscr{S}\left(\mathscr{O}_{2}\right)$ whenever $\mathscr{O}_{1} \supset \mathscr{Q}_{2}$. It is the dual structure to the net of algebras. The equivalence classes at points are the germs, from which we may construct a sheaf whose continuous sections over $\odot$ are the states in $\mathscr{S}(\mathcal{O})$.

The principle of locality tells us that the characterization of theory lies in the description of the germs.

In this we should distinguish two levels which will be exemplified in the next section. The first level which might be called the kinematical level refers to the quantities relevant for the coordination of a germ. The other level gives the restrictions imposed by dynamical laws. 


\section{Models}

We note, not without some surprise, that all successful models in Field Theory follow a common pattern and that on the kinematical level there is no distinction between classical and quantum interpretation.

There is a fiber bundle $B$ whose base space is the 4-dimensional space-time $X$. Over each point in $X$ one has a finite dimensional fiber, the field space. There is no unique, distinguished coordinatization of the fiber but a family of equivalent ones which are related by the structure group $\mathfrak{G}$. The fact that also on base space there is no distinguished coordinatization will not concern us here. There is no intrinsic way to compare points on fibers over different points of $X$ (apart from that of the invariants under $\mathfrak{G}$ ) but there is a "connection" transporting points from one fiber to equivalent points on an infinitesimally close fiber. Functions from $X$ to $\mathfrak{G}$ are called gauge transformations. They change the coordinatization on the fibers and at the same time the coordinatization of the connection. The intrinsic objects consist of a section in the bundle together with a connection. To fix the ideas let us think of the simplest example, the Maxwell-Dirac theory. The fiber is the complex 4-dimensional space of a Dirac spinor $\psi_{\alpha}$. From the 8 real variables one can construct 7 gauge invarints leaving an overall phase factor without intrinsic meaning. The structure group is accordingly $U(1)$. The connection in this bundle is given by the electromagnetic potential $A_{\mu}$ which, by itself, is also unobservable. Among the gauge invariant quantities there are pointlike objects like $\bar{\psi}_{\alpha}(x) \psi_{\beta}(x)$ and the electromagnetic fields $F_{\mu \nu}(x)$ but also line-like objects like $\bar{\psi}(x) \exp i \int_{x}^{y} A_{\mu}\left(x^{\prime}\right) d x^{\mu^{\prime}} \psi(y)$, relevant in the Aharonov-Bohm
effect.

An observable is a gauge invariant map from the set of section-connection pairs to the real numbers.

If we interpret the model as a classical theory we deal with a single sectionconnection pair as the description of a state (in the Heisenberg sense "sub specie aeternitatis"). The dynamical law restricts the admitted section-connection pairs by hyperbolic differential equations. If we interpret the model as a quantum theory then a state is a probability measure for the observables considered as random variables. It may be thought of as a measure over the section-connection pairs (modulo gauge transformations in a coordinatization). There are restrictive laws for the admitted measures. In Feynman's path integral formulation one tries to construct the admitted measures from a single scalar local density, the Lagrangian.

Again we may ask about the rôle of the unobservable quantities in the scheme. If, instead of the bundle described above, we want to use a fiber containing only gauge invariant quantities associated to the base point we need an infinite dimensional fiber involving covariant derivatives of arbitrarily high order. The classical dynamical laws are no longer recognized as a finite, closed system of differential equations. In other words we have to go over from a bundle to a sheaf; the restrictions imposed by the laws concern the germ.

\section{Towards the Specification of a Model within Local Quantum Physics}

According to Sect. 2 we must describe the germs of the presheaf $\{\mathscr{Y}(\circlearrowleft)\}$. For this we may be helped by the observation that finite parts of "phase space" (obtained by restricting both the energy and the spatial extension) should roughly correspond to finite dimensional parts of state space. This requirement on the theory has been 
recognized as essential for several desirable features such as the completeness of the particle picture and good thermodynamic properties. Various formulations, differing in strength and precision have been given $[2,3]$. In the present context we use a version which has unfortunately not been published [4]. Denoting the subset of states in $\operatorname{Rep}_{\mathrm{EP}}$ with energy below $E$ by $\mathscr{J}_{E}$ and their real linear span (base norm space) by $V_{E}$ we consider their restrictions to observables or events in the space-time region $\odot$ and denote them by $\mathscr{S}_{E}(\odot), V_{E}(\odot)$, respectively. Now, if we want to describe an element of $\mathscr{S}_{E}(O)$ up to an accuracy $\varepsilon$ in the norm topology we need (by assumption) a finite number $N(\varepsilon)$ of coordinates, furnished by linearly independent elements of the dual space $V_{E}(\odot)^{*}$. In other words, if $\omega$ and $\omega^{\prime}$ are in $\mathscr{S}_{E}(\odot)$ and

$$
\omega^{\prime}\left(A_{k}\right)=\omega\left(A_{k}\right)(k=1, \ldots, N(\varepsilon)), \quad A_{k} \in V_{E}(\Im)^{*}
$$

then

$$
\left\|\omega^{\prime}-\omega\right\|<\varepsilon .
$$

Let $\Theta_{r}$ be the double cone with the origin as its center and base radius $r$. We need to know how the essential dimension $N(\varepsilon)$ of $\mathscr{S}_{E}\left(\mathscr{O}_{r}\right)$ depends on $\varepsilon$ as $r$ tends to zero and $E$ stays fixed.

We may note here that although we have defined $\mathscr{S}_{E}$ in terms of the states of $\operatorname{Rep}_{\mathrm{EP}}$ the sets $\mathscr{S}_{E}(\odot)$ are common to all physical states due to Remark 3) in Sect. 2. In fact, the bound $E$ for the total energy gives a bound $E^{4}$ for the energy density since by the uncertainty principle we cannot pack this energy into a region of radius below $E^{-1}$.

We conjecture now that in the limit $(r E) \rightarrow 0$ the dimension function $N(\varepsilon)$ must show a very marked behaviour, namely that there is a discrete sequence of positive exponents $0<\lambda_{1}<\lambda_{2} \ldots$ and integers $d_{1}<d_{2} \ldots$ such that

$$
\varepsilon>(E r)^{\lambda_{k}} \text { implies } N(\varepsilon)<d_{k} \text {. }
$$

Then we can introduce a basis in $V\left(O_{r}\right)^{*}$ [in the setting of Sect. 1 this is the self-adjoint part of the algebra $\mathfrak{A}\left(\Theta_{r}\right)$ ], ordered according to relevance for the coordinatization of states of bounded energy in the short distance limit. And we can try to make contact with models such as described in the previous section, looking for a relation of the most relevant coordinates to the coordinates of the fiber and the connection. Here we note that so far we used only that $V\left(G_{r}\right)^{*}$ is a real linear space. If it inherits an algebraic structure from the facial structure of the convex cones $V^{+}\left(O_{r}\right)$ (for instance if there is an embedding in an algebra with complex coefficients) we get relations reminiscent of Wilson's operator product expansion.

Much work has to be done before the value of these suggestions can be assessed. But we can see at least a way how theories within the frame of Local Quantum Physics may be classified.

Acknowledgements. I wish to thank the Research Institute for Mathematical Sciences, Kyoto for the magnificent hospitality and thank Profs. I. Ojima and H. Araki for numerous discussions.

\section{References}

1. Haag, R.: Local quantum physics. Berlin, Heidelberg, New York: Springer (1992)

2. Haag, R., Swieca, J.A.: When does a quantum field theory describe particles. Commun. Math. Phys. 1, 308 (1965)

3. Buchholz, D., Wichmann, E.: Causal independence and the energy-level density of states in local quantum theory. Commun. Math. Phys. 106, 321 (1986)

4. Fredenhagen, K., Hertel, J.: Unpublished manuscript 1979

Communicated by A. Jaffe 